International Journal of Biological Sciences ISSN 1449-2288 www.biolsci.org 2007 3(1):8-11

Short Research Communication

(C) Ivyspring International Publisher. All rights reserved

\title{
Gamma Protocadherin Expression in the Embryonic Chick Nervous System
}

\author{
Kenneth D. Cronin and Anthony A. Capehart \\ Department of Biology, East Carolina University, Greenville, North Carolina 27858, USA \\ Correspondence to: Anthony A. Capehart, Phone: (252) 328-6296, Fax: (252) 328-4178, Email: capehartt@ecu.edu
}

Received: 2006.09.07; Accepted: 2006.10.03; Published: 2006.10.05

Protocadherin $\mathrm{\gamma}(\mathrm{pcdh}-\gamma)$ family expression was examined in the embryonic chick central nervous system by in situ hybridization. Transcripts were visualized in discrete regions of fore-, mid-, and hindbrain at stages 23 and 25 and in spinal cord and optic lobe at stages 27 and 43, respectively. Results suggest that pcdh- $\gamma$ may function cooperatively with other cell adhesion molecules in neuronal differentiation and establishment of neural networks in several areas of the developing brain, particularly regions involved in visual processing.

Key words: cadherin, cell adhesion, central nervous system, chick embryo, neural development, protocadherin

\section{Introduction}

Cadherins are calcium-dependent cell adhesion molecules whose function is critical during vertebrate embryogenesis. A search for additional members of the cadherin superfamily identified the protocadherins [1], integral membrane proteins bearing five or more cadherin-characteristic extracellular repeats and conserved cytoplasmic domains that do not interact directly with $\beta$-catenin as do the classical cadherins [2]. Subsequent discovery of additional protocadherins necessitated grouping into several families, the largest of which is protocadherin $\gamma($ pcdh- $\gamma)$, comprised of 22 clustered genes in human and mouse [3-5]. Genes in the pcdh- $\gamma$ family each possess a single exon encoding the extracellular, transmembrane and a portion of the cytoplasmic domain and all share three small exons encoding the terminal portion of the invariant C-terminal cytoplasmic tail [5]. Expression of different protocadherins in the central nervous system (CNS) has been hypothesized to facilitate neuronal and synaptic organization (reviewed in [6] and [7]) and recent data show that mice null for the entire pcdh- $\gamma$ locus exhibit loss of spinal interneurons and compromised synapse formation $[8,9]$.

Only few studies of protocadherins in the chick developmental model have been reported to date. Expression of the OL-protocadherin family [10-12] and members of the protocadherin a family [13] show a dynamic distribution in the developing avian CNS; however, at present only one published study has identified a member of the pcdh- $\gamma$ family in chick [14] and little is known of pcdh- $\gamma$ expression at early stages of CNS development in this species. Spatial and temporal localization of pcdh- $\gamma$ family expression is therefore an important initial step toward identifying potential function in the avian. To this end, in the present study we investigated pcdh- $\gamma$ family transcript localization during early chick CNS development using a probe recognizing the constant C-terminal cytoplasmic domain.

\section{Materials and Methods}

Samples were fixed in $85 \%$ ethanol, $10 \%$ formaldehyde, $5 \%$ glacial acetic acid, embedded in par- affin and sectioned at $8 \mu \mathrm{m}$. In situ hybridization was performed according to Breitschopf et al. [15] as modified by Capehart and Kern [14]. Briefly, cDNA prepared by RT-PCR from stage 43 [16]) chick brain RNA was utilized to generate a 337 bp cDNA probe template specific for the $\gamma$ protocadherin constant cytoplasmic domain (GenBank accession \# AY325274, bases 3064-3400) using primers 5'-CTTCTCTCAGACCCAGAGAC-3' and 5'-CTTCTTCTCCTTCTTGCCAG-3', which was verified by sequencing and subcloned into pGEMT Easy (Promega). Digoxygenin-labeled RNA probes were prepared from NcoI or NdeI linearized plasmids with the DIG RNA Labeling kit (Roche Molecular Biochemicals) according to manufacturer's instructions and labeled probe quantified by dot blot. Hybridizations were performed $\mathrm{ON}$ at $55^{\circ} \mathrm{C}$ in $2 \mathrm{XSC}$ SS $50 \%$ formamide, $10 \%$ dextran sulfate, $0.02 \%$ SDS, and $0.01 \%$ yeast tRNA containing either $200 \mathrm{ng} / \mathrm{ml}$ antisense or sense (negative control) probes. Sections were washed thoroughly at $55^{\circ} \mathrm{C}$ in 1X SSC and $50 \%$ formamide. Specimens were incubated $1 \mathrm{hr}$ at RT in blocking buffer containing $10 \%$ fetal bovine serum and $1 \%$ sheep serum and $1 \mathrm{hr}$ in 1:1000 sheep anti-digoxygenin-alkaline phosphatase in blocking buffer (Roche). Bound probe was visualized following TBS washes using NBT/BCIP substrate (Roche). CNS structure was identified according to Romanoff [17], LaVail and Cowan [18], and Bellairs and Osmond [19].

\section{Results and Discussion}

The present study reports localization of pcdh- $\gamma$ family mRNA transcripts in chick during the embryonic period in which early differentiation of CNS structure occurs. The region of pcdh- $\gamma$ cytoplasmic domain used as probe template for in situ hybridization represents $100 \%$ sequence identity between the two members of this family in chick present in GenBank at the current time (pcdh- $\gamma$ A5, AY325274 bases 3064-3400 and pcdh- $\gamma C 5$, NM_214674/AB004689 bases 2582-2918). While only two members of the pcdh- $\gamma$ family have thus far been described in chick [14] it is likely that additional pcdh- $\gamma$ genes will be identified as found in other amniotes [3-5]. 
Significant expression of pcdh- $\gamma$ was first detected in the chick CNS at stage 23 particularly within cells along the ventral aspect of the myelencephalon (Figure 1). Little expression of pcdh- $\gamma$ was observed in different regions of the CNS at earlier stages (stages 18 and 21). At stage 23, transcripts were limited to larger cells in the hindbrain, likely neuronal cell bodies, primarily in the mantle zone. Arrangement of pcdh- $\gamma$ labeled cells into identifiable nuclei (e.g., cranial nerve motor nuclei) at this stage could not be ascertained. In mouse, levels of pcdh- $\gamma$ protein were low in adult hindbrain by Western blot [20], but present in the embryonic hindbrain [21] as observed in early chick in the present study. Expression of OL-protocadherin (OL-pcdh), the single member of another of the approximately seventeen different pcdh families described thus far [6, 22], was also reported in the embryonic chick hindbrain [12] with the protein localized predominately in axons along its ventral length. Strong expression of OL-pcdh protein in axon fibers and inability to localize OL-pcdh at cell junctions led to the suggestion that OL-pcdh functions primarily in axon growth or guidance [12] rather than in neuronal differentiation and synaptogenesis as proposed for pcdh-ץ [21].

At stage 25 the chick brain has entered a transitional phase between initial morphogenesis of brain compartments and establishment of the terminal cytoarchitecture comprising nuclei and fiber tracts [17]. Pcdh- $\gamma$ transcripts at stage 25 were largely expressed in the ependymal layer extending into the mantle zone within different sites of the fore-, mid- and hindbrain (Figures 2 and 3) consistent with a role in early differentiation of neuronal subsets. In the telencephalon at this stage of development, mitotic cells of the ependymal layer and incipient neuroblasts of the mantle zone merge over a large area [17] and expression was observed primarily in the dorsal region of the developing cerebrum (pallium). Accumulation of pcdh- $\gamma$ transcripts was particularly strong within the telodiencephalic eminence (Figure $2 b$ ) which merges with the corpus striatum and forms a boundary between the tele- and diencephalon [17]. In the diencephalon, $p c d h-\gamma$ transcripts were also distributed primarily within the dorsal region (future dorsal thalamus and pretectal nuclei) with a notable absence of expression along its ventral length. In the mouse forebrain at mid- to late gestation and in the adult, pcdh- $\gamma$ mRNA expression was shown in different regions, particularly in hippocampus and cerebral cortex $[8,20,21,23,24]$. In chick, OL-pcdh was localized in ventral forebrain at early stages [12] and later associated particularly with visual brain nuclei in areas such as thalamus [11] while the pcdh-a family exhibited moderate and widespread expression in the forebrain of the later stage embryonic chick [13].

Expression of pcdh- $\gamma$ was also observed in basal and alar plate derivatives of the midbrain, the mesencephalic tegmentum and optic tectum, respectively. The tegmentum houses important nuclei, including those of oculomotor and trochlear nerves (cranial nerves III and IV) while at this stage the tectum has initiated formation of the optic lobes [17]. Expression was scattered within the mantle zone of the tegmentum, but more strongly localized in ependymal and adjacent mantle cells of fore- to mid- tectal regions.
Little expression was observed in the posterior aspect of the mesencephalon (Figure 3).

In addition to earlier localization in the myelencephalon, pcdh- $\gamma$ transcripts were also weakly expressed at stage 25 in the dorsal metencephalon (future cerebellum) as shown in Figure 3. Little signal observed in the future pons. Localization of chick OL-pcdh [12], chick pcdh-a [13] and mouse pcdh- $\gamma$ [20, 21] in the cerebellum at later embryonic, postnatal and adult stages has been reported previously, although other accounts of pcdh expression in the early stage metencephalon are lacking.

In the developing spinal cord at stage 27, pcdh- $\gamma$ family transcripts were present, particularly in the dorsal horn and floorplate regions (Figure 4a-c). Expression was also observed in dorsal root ganglia (DRG). Expression in the dorsal horn and DRG is consistent with that reported for a single member of the pcdh- $\gamma \mathrm{A}$ subfamily in chick, $\gamma \mathrm{A}$-like pcdh (pcdh- $\gamma \mathrm{A} 5 ;$ [14]), and suggests a role for one or more members of the pcdh- $\gamma$ family in interaction between interneurons and the DRG during establishment of peripheral sensory connections with the CNS. In embryonic mouse spinal cord, expression of the pcdh- $\gamma$ family was widespread in gray matter, but preferentially associated with the dorsal horn in the adult [20] and appropriate spinal cord interneuron survival and synapse formation or maintenance have been demonstrated dependent on the pcdh- $\gamma$ family $[8,9]$. In addition, pcdh- $\gamma$ protein was demonstrated in early axonal and dendritic growth cones of cultured murine e12.5 spinal cord neurons, later becoming progressively restricted to the somatodendritic compartment indicating that pcdh- $\gamma$ members may also participate in neuronal maturation and neurite outgrowth [20]. Expression in the chick floorplate as seen with the pcdh- $\gamma$ constant region in situ hybridization probe used in the present study was not observed earlier for $\gamma$ A-like pcdh [14], but in mouse, pcdh- $\gamma$ isoforms and pcdh 15 have been reported in floorplates of brain ventricles [24] and the neural tube [25]. The difference in chick neural tube localization between the common $\gamma$ family probe used in the present study and isoform specific $\gamma$ A-like pcdh probe reported previously supports the notion that more than one pcdh- $\gamma$ family member is expressed in the developing chick spinal cord. Interestingly, chick OL-pcdh is found only in the motor column of the ventral horn [12] further suggesting that multiple pcdh families may function in different spinal cord compartments.

The optic lobes represent a very highly developed part of the chick brain and the visual pathways that arise therein from receipt of retinal afferents and projection of efferents to the thalamus and other forebrain areas are largely responsible for avian visual acuity $[11,17,18]$. Because of strong expression noted during early differentiation of the optic tectum, we also examined pcdh- $\gamma$ family expression in the optic lobe of the later stage 43 embryo (Figure 5). At stage 43 , pcdh- $\gamma$ was highly expressed in multiple laminae of the stratum griseum and fibrosa superficiale (SGFS, Figure 5a) and particularly within thalamic projection neurons of the stratum griseum centrale (SGC, Figure $5 a$ and $b)$. Indeed, in the late embryonic stage chick optic lobe, yA-like pcdh (pcdh-үA5; [14]), pcdh-a [13] and OL-pcdh [11] were also expressed in a pattern 
similar to that seen for the pcdh- $\gamma$ in the present study, consistent with the suggestion that multiple pcdh families are involved in the complex adhesive interactions necessary for neuronal organization and interconnection during establishment of chick visual circuitry.

\section{Conclusion}

In summary, the present study has reported localization of pcdh- $\gamma$ family mRNA transcripts at multiple, distinct sites in the avian CNS. The pcdh- $\gamma$ expression pattern suggests a role in neuronal differentiation and in important functions such as visual processing, where the pcdh- $\gamma$ family may work cooperatively with other members of the cadherin superfamily to ensure appropriate establishment of neuronal connections during chick development.

\section{Conflict of Interests}

The authors have declared that no conflict of interest exists.

\section{References}

1. Sano K, Tanihara H, Heimark RL, et al. Protocadherins: A large family of cadherin-related molecules in central nervous system. EMBO J. 1993; 12:2249-2256.

2. Suzuki ST. Recent progress in protocadherin research. Exp Cell Res. 2000; 261:13-18.

3. $\mathrm{Wu} \mathrm{Q}$, Maniatis T. A striking organization of a large family of human neural cadherin-like cell adhesion genes. Cell. 1999; 97:779-790.

4. $\mathrm{Wu} Q$, Maniatis T. Large exons encoding multiple ectodomains are a characteristic feature of protocadherin genes. Proc. Natl. Acad. Sci. 2000; 97:3124-3129.

5. Wu Q, Zhang T, Cheng J-F, et al. Comparative DNA sequence analysis of mouse and human protocadherin gene clusters. Genome Res. 2001; 11:389-404.

6. Frank M, Kemler R. Protocadherins. Curr. Opinion Cell Biol. 2002; 14:557-562.

7. Junghans D, Haas IG, Kemler R. Mammalian cadherins and protocadherins: about cell death, synapses and processing. Curr Opinion Cell Biol. 2005; 17:1-7.

8. Wang $X$, Weiner JA, Levi $S$, et al. $\gamma$-protocadherins are required for survival of spinal neurons. Neuron. 2002; 36:843-854.

9. Weiner JA, Wang X, Tapia JC, et al. Gamma protocadherins are required for synaptic development in the spinal cord. Proc. Nat. Acad. Sci. 2005; 102:8-14.

\section{Figures}

Figure 1. Pcdh- $\gamma$ in the stage 23 hindbrain. (a) Pcdh- $\gamma$ transcripts are localized primarily within the mantle zone (arrow). Anterior-most neural tissue on the right side of panel is slightly posterior to the midbrain/hindbrain boundary. The hindbrain roofplate is indicated by the arrowhead. Boxed area of mantle zone is shown in higher magnification in (b). (c) Corresponding region of ventral hindbrain shows lack of signal with sense control probe. Scale bars $=250 \mu \mathrm{m}$ for panels $1 \mathrm{a} \& \mathrm{c}$ and $25 \mu \mathrm{m}$ for $1 \mathrm{~b}$.

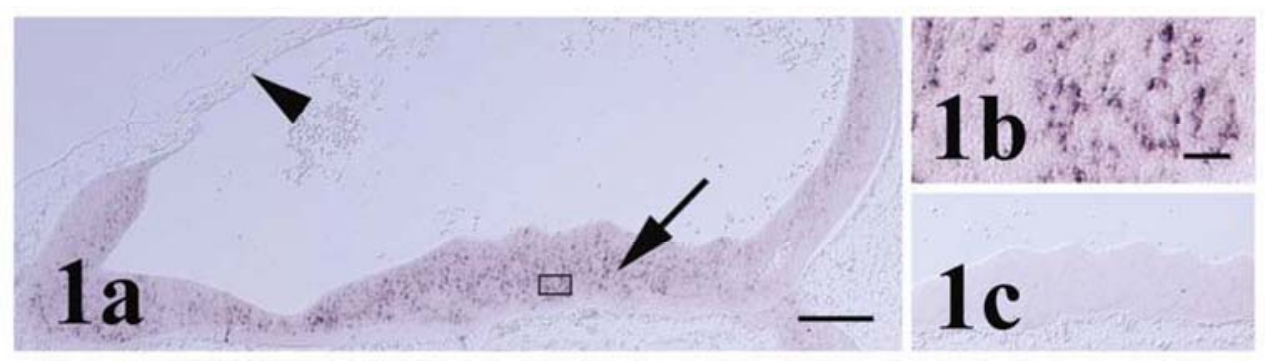


Figure 2. Pcdh- $\gamma$ in the stage 25 fore- and midbrain. (a) Pcdh- $\gamma$ expression is observed in dorsal telencephalon (T, small arrow), diencephalon (D, large arrow), and mesencephalon (M, arrowhead). Signal is also observed in the mesencephalic tegmentum (large asterisk). Little staining is observed in the ventral forebrain surrounding the optic recess (small asterisk) and optic chiasm (small arrowhead). Boxed region of telodiencephalic eminence shows strong signal in cells at higher magnification in (b). (c) Control section shows lack of signal in telodiencephalic eminence at this stage. Scale bars $=250 \mu \mathrm{m}$ for panels $2 \mathrm{a} \& \mathrm{c}$ and $10 \mu \mathrm{m}$ for $2 \mathrm{~b}$.

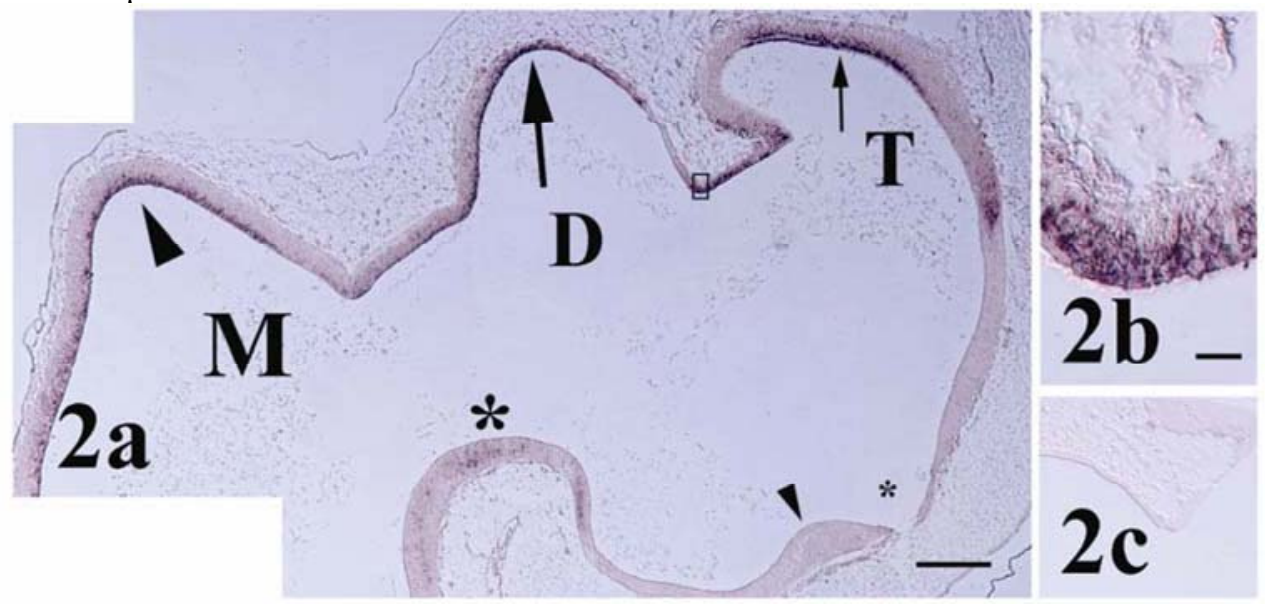

Figure 3. Pcdh- $\gamma$ in the dorsal metencephalon (arrow) at stage 25. Separation of future pons and cerebellum is marked by the isthmus (asterisk). Scale bar $=250 \mu \mathrm{m}$.

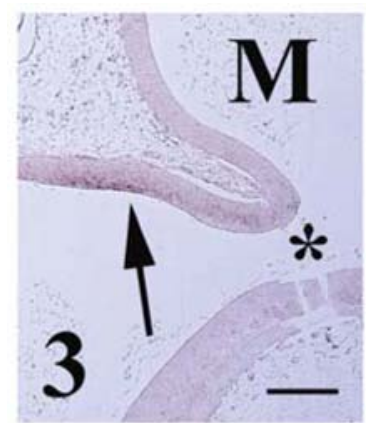

Figure 4. Pcdh- $\gamma$ in the stage 27 spinal cord. (a) Localization of pcdh- $\gamma$ transcripts in the dorsal horn, floorplate (arrow) and dorsal root ganglion (arrowhead). Staining of interneurons in the developing dorsal horn (boxed area) is shown in higher magnification in (b). Higher magnification image of floorplate pcdh- $\gamma$ expression is shown in (c). (d) Section incubated with control probe shows lack of signal in spinal cord and dorsal root ganglia. Scale bars $=250 \mu \mathrm{m}$ for panels $4 \mathrm{a} \& \mathrm{~d}, 25 \mu \mathrm{m}$ for $4 \mathrm{~b}$ and $50 \mu \mathrm{m}$ for $4 \mathrm{c}$.
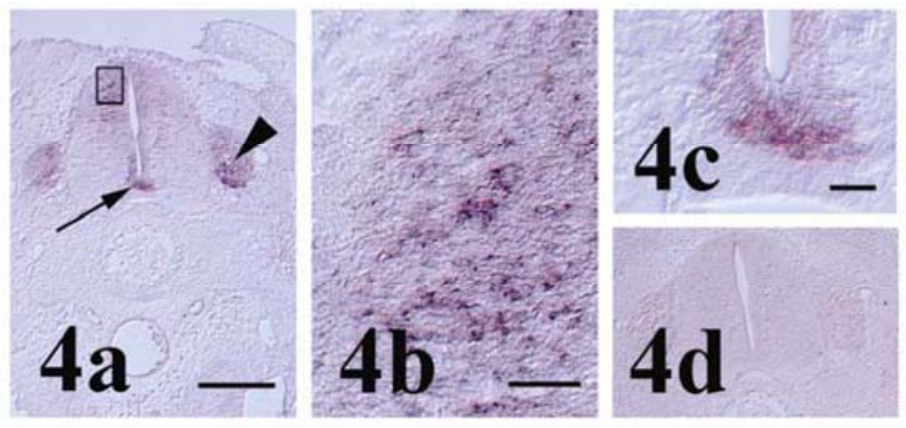

Figure 5. Pcdh- $\gamma$ in the stage 43 optic lobe. (a) Pcdh- $\gamma$ is expressed in multiple laminae of the stratum griseum and fibrosa superficiale (SGFS) and in neurons of the stratum griseum centrale (SGC). Boxed region of SGC at higher magnification in (b) shows strong localization in neuronal cell bodies. Scale bars $=250 \mu \mathrm{m}$ for panel $5 \mathrm{a}$ and $25 \mu \mathrm{m}$ for $5 \mathrm{~b}$.
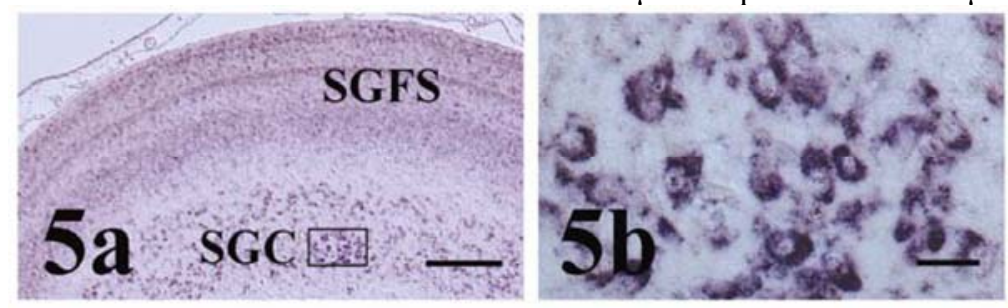